This item was submitted to Loughborough's Research Repository by the author.

Items in Figshare are protected by copyright, with all rights reserved, unless otherwise indicated.

\title{
Epstein-Barr Virus
}

\section{PLEASE CITE THE PUBLISHED VERSION}

https://doi.org/10.1002/9780470015902.a0001020.pub3

\section{PUBLISHER}

Wiley

\section{VERSION}

AM (Accepted Manuscript)

\section{PUBLISHER STATEMENT}

This work is made available according to the conditions of the Creative Commons Attribution-NonCommercialNoDerivatives 4.0 International (CC BY-NC-ND 4.0) licence. Full details of this licence are available at: https://creativecommons.org/licenses/by-nc-nd/4.0/

\section{LICENCE}

CC BY-NC-ND 4.0

\section{REPOSITORY RECORD}

Morris, Mhairi. 2019. “Epstein-barr Virus”. figshare. https://hdl.handle.net/2134/27474. 

postgraduates, and researchers reading outside their field of expertise.)

\section{Abstract}

Epstein-Barr virus (EBV) is a ubiquitous gamma herpesvirus aetiologically linked to different lymphoid and epithelial malignancies, and a number of systemic autoimmune diseases. The virus has a unique ability to transform resting $B$ lymphocytes in vitro by expressing a set of latent genes, subsets of which are present in EBV-associated tumours. EBV exploits the physiology of normal B-cell differentiation to persist within the memory $B$ cell pool of the immunocompetent host with strong T-cell responses important for controlling EBV infection. Immunosuppressed transplant recipients and human immunodeficiency virus (HIV)-infected individuals are at increased risk of developing EBV-transformed B-cell proliferations which often present as monoclonal nonHodgkin lymphomas. The major EBV-associated tumours (Burkitt lymphoma, Hodgkin lymphoma and nasopharyngeal carcinoma) show restricted forms of latent viral gene expression reflecting a more complex pathogenesis involving additional cofactors. A number of pharmacological and immunotherapeutic approaches are being developed to treat or prevent these EBV-associated tumours.

\section{Key concepts}

- Epstein-Barr virus (EBV) infection is implicated in the aetiology of several different lymphoid and epithelial malignancies, as well as a number of systemic autoimmune diseases.

- EBV exploits the physiology of normal B-cell differentiation to persist within the memory B cell pool of the immunocompetent host.

- EBV-encoded latent genes induce B-cell transformation in vitro by altering cellular gene transcription and constitutively activating key cell signalling pathways.

- Immunosuppressed transplant patients are at risk of developing EBV-transformed B-cell proliferations presenting as B-cell lymphomas.

- Other EBV-associated tumours display more restricted forms of latent gene expression, reflecting more complex pathogenesis involving additional cofactors.

- EBV sequence variation may reflect disease risk.

- Pharmacological and immunotherapeutic approaches are being developed to treat or prevent EBVassociated tumours.

- More direct vaccine approaches are being examined for the treatment and prevention of EBVassociated diseases.

\section{Introduction: Discovery of Epstein-Barr Virus}


Denis Burkitt. An Irish surgeon stationed at a mission hospital in rural Uganda, he described for the first time a paediatric malignancy that usually presented as a fast-growing tumour of the jaw. The afflicted children came from geographically distinct regions of Uganda. His curiosity piqued, Burkitt set out to visit hospitals in 10 African countries, in an attempt to correlate the incidence of the tumour with other environmental factors. He discovered that the tumour was found in an area overlapping the malaria belt (Burkitt, 1962). Suspecting that a mosquito-borne virus might be causing the disease, Burkitt presented his findings in a seminar at the Middlesex Hospital in London in 1961. See also Leukaemias and Lymphomas, and $\underline{\text { Non-Hodgkin Lymphomas }}$

Attending that lecture was the next medical detective on the case, M Anthony Epstein, a pathologist. He knew that, while a large number of viruses were known to cause cancer in animals, none of these was mosquito-borne. Nonetheless, for the next 3 years, he and his colleagues in London, Bert Achong and Yvonne Barr, examined biopsies of the tumour now called Burkitt lymphoma (BL), looking for evidence of a human tumour virus. A technical breakthrough came in 1964 with the establishment of several BL cell lines, from which herpesvirus-like particles were finally visualized by electron microscopy (Figure 1, Epstein et al., 1964). None of the three herpesviruses known at that time (Herpes simplex virus 1 and 2 and Varicella-Zoster virus) was lymphotropic, nor were they associated with cancer or transmitted by mosquitoes. See also Herpesviruses (Human), and Oncogenic Viruses

\section{<Figure 1 near here>}

7

Nevertheless, now that the existence of a virus was confirmed, Werner and Gertrude Henle took up the investigation in Philadelphia, using immunological techniques to determine whether this African tumour-associated virus was in fact new. They found that the BL patients did indeed have antibodies that specifically recognized an antigen in the cultured tumour cells, and did not react with any of the three known herpesviruses in infected cells. Harald zur Hausen, a German scientist working with the Henles, also detected EBV deoxyribonucleic acid (DNA) in a BL cell line and in biopsies of nasopharyngeal carcinoma (NPC; zur Hausen et al., 1970). It appeared that the suspect, a new human herpesvirus now called EBV, had been identified.

At this point the Henles were surprised to discover that whereas all the African BL patients had anti-EBV antibodies, as did most of the normal population in the USA, no disease had been associated with the infection in the USA. Subsequently the Henles' research technician, who was among the relatively rare adults with no EBV antibodies, contracted infectious mononucleosis (IM). On her return to the laboratory, not only did she have EBV antibodies, but her B lymphocytes reacted with antibodies from BL patients, and when explanted to cell culture, grew continuously as an immortalized cell line, whereas before the infection they did not (Henle et al., 1968). This serendipitous discovery identified EBV as the causative agent for IM, later proven in a seroepidemiological study of university students and by molecular hybridization analyses designed to identify EBV in saliva. See also Infectious Mononucleosis

In the late 1960s, the ability of EBV to infect and immortalize human B lymphocytes in culture was discovered principally by John Pope working in Australia. This property singles EBV out as the most efficient transforming agent for human cells 
in culture and still provides a useful tool for rescuing the human genome for genetic and functional studies. Since this time EBV has been implicated in the aetiology of an increasing number of tumours (B- and T-cell lymphomas, Hodgkin lymphoma (HL), a proportion of gastric adenocarcinomas, and although less commonly, a subset of breast cancers and a rare childhood cancer of the smooth muscle system called leiomyosarcoma). There are several associations of EBV with the pathogenesis of other diseases, such as oral hairy leukoplakia (OHL), chronic fatigue syndrome, and certain systemic autoimmune diseases including multiple sclerosis, rheumatoid arthritis, systemic lupus erythematosus (SLE) and systemic scleroderma (SSc), but for some of these diseases, the exact role of EBV infection in their pathogenesis has yet to be defined. See also Electron Microscopy, Epstein-Barr Virus and Cancer, Nucleic Acids: Hybridisation, and Human Autoimmune Diseases.

\section{Pathogenesis and Epidemiology}

\section{Primary and persistent infection}

The hallmark of herpesvirus infection is the ability to establish a quiescent, nonproductive infection that persists, often silently, for life. The cell type permitting this long-term latent infection varies, with Herpes simplex virus (HSV) persisting in neurons, Cytomegalovirus (CMV) in secretory glands, kidneys and other tissues, and EBV in B lymphocytes. It is generally believed that EBV replicates and is shed at epithelial sites in the oropharynx and salivary glands but that B lymphocytes are the main site of EBV persistence. This is supported by the B lymphotropism of EBV which is mediated by the binding of the major viral envelope glycoprotein gp350/220 to the CR2 (CD21) receptor on the surface of B cells. Since epithelial cells lack the $\mathrm{CR} 2$ receptor, the virus-encoded fusion proteins, $\mathrm{gB}$ and $\mathrm{gH} / \mathrm{gL}$ are the key mediators of EBV entry into the epithelial compartment (Chen et al., 2016).

Detailed examination of EBV infection in vivo has shown that the virus persists in the $\operatorname{IgD} \mathrm{CD}_{2} 7^{+}$memory $\mathrm{B}$ cell subset and that these cells have downregulated the expression of most, if not all, viral genes (Babcock et al., 1998). This reservoir of infected cells is stably maintained thereafter, apparently subject to the same physiologic controls as the general mucosaassociated memory B cell pool. EBV persistence within this B-cell population brings with it the possibility of fortuitous antigen-driven recruitment of infected cells into germinal centres, leading to progeny that either reenter the circulating memory pool or differentiate to plasma cells that may migrate to mucosal sites (Figure 2). The different forms of latency that are manifest in virus-associated malignancies (discussed later) may represent latency programmes that have evolved to accommodate such changes in host cell physiology.

\section{<Figure 2 near here $>$}

The majority of the global population is infected subclinically during childhood. EBV-induced symptoms vary with the age of infection and whether the infection is primary or reactivated. The human diseases associated with EBV infection are summarized in Table 1 . In developing countries, most infants are infected asymptomatically within the first year of life, although they are protected for the first 3 months by maternal antibodies. In upper socioeconomic classes, infection usually occurs within the first three decades, which may manifest in adolescents and young adults as infectious mononucleosis (IM). 
EBV elicits both humoral and cell-mediated immune responses in infected hosts. The levels of EBV-specific antibodies against latent and lytic antigens remain fairly stable in healthy EBV-positive individuals but are elevated in different EBVassociated diseases (see later discussion). As with other persistent viruses, cell-mediated immunity plays an important role in controlling EBV infection with high levels of virus-specific $\mathrm{CD} 8^{+}$and $\mathrm{CD} 4^{+} \mathrm{T}$ cells being detectable in the circulation. See also Epstein-Barr Virus and Cancer

\section{<Table 1 near here>}

\section{B-cell (immunoblastic) lymphomas in the immunosuppressed}

EBV-induced lymphoproliferative syndromes resulting from primary, or later from reactivated, infection arise in immunodeficiency states, because virus-induced B-cell proliferation is unchecked by EBV-specific T cells. Up to $10 \%$ of allograft recipients are affected, depending on the intensity of immunosuppressive therapy. Primary immunodeficiency states, whilst themselves rare, have a very high incidence of EBV-induced B-cell lymphomas; these include X-linked lymphoproliferative syndrome (Duncan syndrome), Wiscott-Aldrich syndrome, ataxia telangiectasia and combined immunodeficiency disease. These EBV-induced proliferations, initially polyclonal, express B-cell surface markers and do not have distinctive chromosomal abnormalities but can evolve into aggressive non-HLs. These lesions initially resemble the lymphoblastoid cell lines (LCLs) generated in vitro by infection of B lymphocytes with EBV (Young et al., 1989). Until recently the majority of these tumours presented in the context of human immunodeficiency virus (HIV) infection but with the introduction of highly active anti-retroviral therapy the incidence of these lesions has diminished. See also AIDS: $\underline{\text { Clinical Manifestations, Immunodeficiency, Infections in the Secondary Immunocompromised Host, and Immunodeficiency, }}$ Primary: Affecting the Adaptive Immune System

\section{Burkitt lymphoma and Hodgkin lymphoma}

The first lymphoproliferative disease associated with EBV was BL, the most common childhood cancer in the endemic areas of sub-Saharan Africa, with an incidence of 10 per 100000 during the first 15 years of life (Thorley-Lawson and Allday, 2008). Although clonal EBV genomes are present in almost all African BL, they are mostly absent in the sporadic form, which occurs, rarely, in Europe and North America. For this reason and because of the role of the c-myc proto-oncogene (thought to be responsible for the neoplasm), EBV is now viewed as a nonessential cofactor. The definitive marker for BL, regardless of geographical origin and EBV positivity, is a set of specific chromosomal translocations. These result in the reactivation of the c-myc proto-oncogene at chromosome $8 \mathrm{q} 24$ by placing it adjacent to the immunoglobulin regulatory elements on chromosomes 14, 22 or 2 (Dalla-Favera et al., 1982). Higher EBV titres among children in the endemic area indicate increased risk for developing BL; however, EBV probably contributes as a cofactor by stimulating B-lymphocyte proliferation, increasing the likelihood of selection of cells bearing the c-myc translocation. 


\section{EBV-associated T- and NK-cell lymphomas}

EBV infection is also associated with approximately $40 \%$ of HL cases where both viral DNA and latent proteins are localized to the malignant component of the tumour, the so-called Hodgkin and Reed-Sternberg (HRS) cells. The association of HL with EBV is age-related; paediatric and older adult cases are usually EBV-associated whereas HL in young adults is less frequently virus-positive. Although the incidence of HL is relatively low (1-3/100 000 per year), this tumour is not geographically restricted making its association with EBV significant in world health terms. See also Chromosome $\underline{\text { Rearrangements, }}$ and Oncogenes

EBV-positive monoclonal lymphomas of either $\mathrm{CD} 4^{+}$or $\mathrm{CD} 8^{+} \mathrm{T}$ cell origin are more frequently found in Southeast Asian populations arising as a consequence of either virus-associated haemophagocytic syndrome (VAHS) or in the setting of chronic active EBV infection. A more aggressive extranodal tumour predominantly expressing the CD56 NK marker is also EBV-positive and manifests as an erosive lesion (lethal midline granuloma) of the nasal cavity.

\section{Nasopharyngeal carcinoma and gastric adenocarcinoma}

The epithelial malignancy NPC, like BL, is characterized by distinct patterns of geographical distribution. It is particularly common in areas of China and South-East Asia, reaching a peak incidence of 20-30 cases per 100000 per year and also occurs with intermediate incidence among Alaskan Inuit people and in several pockets in North Africa. Incidence rates are particularly high in Cantonese males highlighting an important genetic predisposition as well as a role for environmental cofactors such as dietary components (e.g. salted fish). Arising in the posterior nasopharynx in the Waldeyer ring, the peak age for NPC incidence is 40-60 years, mainly in males. The association between EBV and NPC was first suspected from serological studies, as NPC patients carried extremely high titres of antibodies directed against the EBV early and viral capsid antigens (EA/VCA). These elevated titres may appear before the tumour develops, a discovery with enormous public health implications in the endemic area. Widespread EBV antibody screening in parts of Guangdong and Guangxi provinces in southern China, especially for IgA antibodies against VCA, has significantly increased the NPC early detection rate. Recent studies have demonstrated that the quantitative analysis of tumour-derived EBV DNA in the blood of patients with NPC using real-time polymerase chain reaction (PCR) is of both diagnostic and prognostic utility (Chan et al., 2002).

The clonal EBV genome is detected in all cells of undifferentiated NPC tissue specimens, regardless of geographic origin, as well as in premalignant lesions of the nasopharynx (Raab-Traub and Flynn, 1986), but only a handful of EBV latency genes are expressed. NPC tumours are also characterized by a prominent lymphoid stroma and the interaction between these activated lymphocytes and adjacent carcinoma cells appears to be crucial for the continued growth of the malignant component. An aetiologically complex disease, NPC has been linked to several dietary, environmental and genetic cofactors. Several EBV-inducing tumour-promoting agents have been identified as environmental cofactors in the high-incidence areas. Detailed analysis of the genetic changes in NPC suggests that some of these (particularly deletions in chromosomes $3 p$ and 9p) occur early before EBV infection (Lo and Huang, 2002). Thus, EBV may be a tumour promoting agent in NPC and 
one possibility is that the genetic changes induced by carcinogen exposure create a cellular environment in which EBV latency can be aberrantly established leading to the outgrowth of transformed cells. See also Cancer, $\underline{\text { Cancer Cytogenetics, }}$ Cytogenetic Techniques, Environmental Carcinogens and Mutagens, and Tumour Suppressor Genes

EBV infection is also present in approximately $10 \%$ of typical gastric adenocarcinomas, accounting for up to 75000 cases per year. These tumours resemble NPC in carrying monoclonal EBV genomes, having a restricted pattern of EBV gene expression and in the appearance of virus infection as a relatively late event in the carcinogenic process. The geographical variation in the association of EBV with gastric adenocarcinomas probably reflects ethnic and genetic differences. EBVpositive tumours have distinct phenotypic and clinical characteristics compared with EBV-negative tumours. See also Epstein-Barr Virus and Cancer

\section{Rarer EBV-associated tumours}

\section{Breast cancer:}

Albeit controversial, EBV infection has also been linked to breast cancer incidence, with approximately $30-40 \%$ of breast cancers exhibiting EBV latent gene product expression (Labrecque et al., 1995; Ballard, 2015). By contrast, other studies failed to detect EBV gene expression in breast cancer tissues (Fuentes-Pananá et al., 2016). To this day, the nature of the relationship between EBV infection and breast cancer is poorly understood and remains controversial, although evidence points to an association of EBV infection with more aggressive forms of breast cancer (Mazouni et al., 2011). Moreover, it is believed there is a correlation between EBV-positivity in breast cancer with mutations in the APOBEC family of cytodine deaminases - enzymes which ordinarily serve to protect against viral infections (Hu et al., 2016).

\section{Leiomyosarcoma:}

Leiomyosarcomas are extremely rare, differentiated smooth muscle tumours that are associated with EBV infection, primarily in immunocompromised children, such as transplant recipients, children with congenital immunodeficiencies, and HIV patients (Lee et al., 1995; Tetzlaff et al., 2011). The presence of the EBV-encoded RNAs (EBERs) in the smooth muscle tissue suggests that EBV is capable of infecting smooth muscle cells in addition to its normal tissue tropism in B cells and epithelial cells, findings which are supported by the evidence that the EBV receptor, CR2 (CD21), is also present on these cells (McClain et al., 1995).

\section{Oral hairy leukoplakia}

Oral hairy leucoplakia (OHL), a benign lesion of the tongue, may be the only EBV-associated disease whose pathology is a direct result of full-blown viral replication (Greenspan et al., 1985). OHL occurs in up to $25 \%$ of patients with acquired immunodeficiency syndrome (AIDS) and in other immunosuppressed individuals, and can contain multiple strains of EBV. EBV episomes are not detected, and there are masses of EBV particles in the lesions. The lesions respond well to the antiviral drug acyclovir. 


\section{EBV-associated autoimmune disorders}

2

A number of genetic and environmental factors are involved in the aetiology of systemic autoimmune diseases, including dysregulation of EBV. The restricted pattern of gene expression observed in latent EBV infection in memory B-cells enables the virus to evade the host immune defence, which may also lead to initiation of autoimmune diseases or exacerbate disease progression. Common features of EBV-associated autoimmune disorders such as Multiple Sclerosis (MS), Rheumatoid Arthritis (RA) and Systemic Lupus Erythematosus (SLE) include elevated titres of EBV antibodies, reduced anti-EBV T cell responses, and increased viral load.
The mechanisms underlying the causal role of EBV in promoting disease onset or progression remains poorly understood. For example, in MS, it is generally believed that causation is multifactorial, with EBV infection or reactivation representing one of many causal factors, alongside genetic defects in the immune response to EBV infected B cells, vitamin D deficiency and molecular mimicry between viral peptides and host epitopes, leading to an autoimmune attack on host cells and their components (Tao et al., 2017).

\section{Classification and Structure}

Sharing nearly indistinguishable morphology, herpesvirus virions are all enveloped, with an amorphous protein tegument region encasing a 162-capsomer icosahedral capsid. All have double-stranded linear DNA genomes, and, based on their biological properties, are classified into three subfamilies. EBV and Human herpesvirus 8 (HHV-8) are the only members of the Gammaherpesvirinae to infect humans routinely. Most likely the causative agent of AIDS-related Kaposi's sarcoma, HHV-8 has an endothelial cell tropism, and shares EBV's B-cell tropism. See also Herpesviruses (Human)

The 184-kb EBV genome is characterized by distinctive sequence reiterations. At both termini are $2-5$ copies of a 500-bp direct repeat unit (the terminal repeat, TR), through which the linear genome circularizes to form the episome, the molecular basis for latent infection (Figure 3). The genome structure and organization of EBV are most homologous with the other Old World primate members of its genus Lymphocryptovirus (LCV), distinguished by their lymphotropism and propensity to oncogenicity. A transforming, EBV-related virus has also been isolated from spontaneous B-cell lymphomas of common marmosets and is thus the first EBV-like virus to be identified in New World primates.

\section{Virology}

The EBV genome assumes different forms, depending on the state of infection. During latency, it exists as a chromatinassociated episome with a varying number of TR units for different clonal isolates. Using host-cell DNA polymerase, each EBV episome is replicated during the cell cycle, maintaining a constant copy number from one cell generation to the next. Very few of EBV's 100-odd genes are expressed during latency, all of them localizing to either the nucleus or membrane of the infected cell. EBNA1 is the only virally encoded gene required for persistence of the episome (Yates et al., 1985), and is the only EBV protein expressed in all EBV-associated malignancies. A protein of low abundance, EBNA1 maintains EBV 
latency by binding as a homodimer in a highly sequence-specific manner to a series of repeat units comprising the EBV origin of plasmid replication, ori-P. A unique structural feature of EBNA1 is the presence of an internal Gly Gly Ala repeat unit of varying length which serves to prevent recognition of this protein by cytotoxic T lymphocytes (CTLs; Levitskaya et al., 1997). This may contribute to the ability of EBV to persist in memory B cells (Figure $\underline{3}$ ). See also Immune Response: Evasion and Subversion by Pathogens, and T Lymphocytes: Cytotoxic

\section{Sequence variation}

The first complete EBV sequence to be published was the B95.8 laboratory strain in 1984 (Baer et al., 1984), which at the time was the largest DNA sequence to have been determined and made up 10\% of the EMBL data library. This strain of EBV was isolated from the only EBV cell line available at the time that produced sufficient levels of virus to enable cloning of the viral restriction fragments; however over the years it has become apparent that there are multiple strains of the virus worldwide. Broadly speaking, they can be categorised into Type 1 and Type 2 - a classification system based on the gene sequence of EBV nuclear antigens 2 and 3 (EBNA2 and EBNA3; Tzellos and Farrell, 2012). Type 2 is common in parts of Africa, whilst Type $1 \mathrm{EBV}$ is the most prevalent strain globally and is more efficient at transforming B cells into LCLs in vitro. It has long been hypothesised that the genetic variation in EBV strains across different geographical regions may account for the substantial geographic differences in EBV-associated disease incidence (Jenkins and Farrell, 1996). In support of this hypothesis is the recent findings from Tsai et al., (2017), which mapped out the different biological properties of particular EBV strains to explain their association with different EBV-associated cancers. For example, the YCCEL1 EBV strain isolated from a gastric carcinoma and the M81 strain isolated from a nasopharyngeal carcinoma both infected primary epithelial cells, gastric carcinoma cells and gastric spheroids more efficiently than the well-characterised Akata or B95.8 laboratory strains (Tsai et a1., 2017). Furthermore, interestingly there is a high frequency of co-infection with both Type 1 and Type 2 virus strains in MS patients, suggesting a synergistic or increased risk association in individuals harbouring more than one virus strain, presumably having undergone more than one primary infection event (Santón et al., 2011). In addition to this broad distinction between EBV Types 1 and 2, there is also minor heterogeneity within each virus type particularly within the latent membrane protein 1 (LMP1) gene. Although strong biological evidence is lacking, it remains possible that variation in EBV genes within different virus strains can contribute to the risk of developing virusassociated tumours.

\section{Viral Gene Expression during EBV Latency}

Only a small fraction of the genes encoded by EBV is expressed during latency because transcription in latently infected cells is tightly regulated and restricted to a small portion of the genome. Four latency types are distinguished by the number of EBV latency proteins made. They range from the EBNA1-only type I latency found in the majority of BL tumours to type III latency in EBV-transformed LCLs, in which all of the known latent genes are expressed. Latency type 0 is characterised by the lack of all viral gene expression observed in non-dividing B cells. The gene expression and viral promoter usage 
characteristic of type III latency is accompanied by a lymphoblastoid pattern of cellular gene expression, which includes high levels of the B-cell activation antigens and cellular adhesion molecules. The first proteins expressed after primary EBV infection of B lymphocytes in vitro, which results in type III latency, are EBNA2 and EBNA-LP (leader protein). Within $48 \mathrm{~h}$ the other EBNA genes, including EBNA1 and a pair of nonpolyadenylated ribonucleic acid (RNA) polymerase III transcripts, the Epstein-Barr-encoded RNAs (EBERs), are expressed. The transcripts encoding all of the EBNAs are highly spliced, and can arise from either of the two promoters, Wp and Cp (Figure $2 \mathrm{a}$ ). Concomitant with EBNA expression, latently infected cells also express two membrane proteins: (i) LMP1, EBV's principal oncoprotein and (ii) LMP2A and LMP2B which regulate virus latency The promoters for the LMP proteins are EBNA-2 responsive and are probably coordinately regulated. Thus the full range of latency genes plus the EBERs are expressed in type III latency as observed in LCLs and EBV-induced lymphoproliferations in vivo.

At the other extreme is type I latency which is observed in fresh BL biopsies, with expression of EBNA1 and the EBERs only. In this situation EBNA1 expression is driven from a different promoter (Qp) compared to that used in type III latency $(\mathrm{Cp} / \mathrm{Wp})$. This differential promoter usage is regulated by epigenetic modification of various EBV promoters. Recently, a novel form of EBV latency has been found in some BL biopsies where deletion of EBNA2 has occurred and EBNA expression is driven from the Wp promoter (Kelly et al., 2002). Type II latency is observed in NPC, and HL is restricted to expression of EBNA1 along with LMP1 and LMP2A/B. However, LMP1 expression at the protein level is only observed in encoded protein 1 (BARF1).

\section{Function of Key EBV Latent Genes}

Several of the latent gene products exert profound effects on cellular gene expression, perturbing the network of cellular factors controlling cell growth. EBNA2 is a transcription factor, activating an array of viral and cellular genes. It does not directly bind DNA but is targeted to specific DNA sequences by the cellular protein RBP-JК (recombination signal sequence-binding protein-J kappa), and is associated with other cellular proteins within the transcription complex (Ling et al., 1993). Interestingly EBNA2 is functionally homologous to the Drosophila Notch-1 factor. See also Drosophila Patterning: Delta-Notch Interactions

Apart from its effect in the replication and maintenance of the episomal EBV genome, EBNA1 may also contribute directly to oncogenesis via the modulation of various cell signalling pathways. Thus, EBNA1 has been shown to promote survival in BL cells and, via interaction with a cellular ubiquitin-specific protease, USP7 or HAUSP, promotes disruption of PML (promyelocytic leukaemia protein) nuclear bodies thereby affecting the efficiency of DNA repair.

LMP1 is the major transforming protein of EBV behaving as a classical oncogene in rodent fibroblast transformation assays and being essential for EBV-induced B-cell transformation in vitro (Wang et al., 1985). LMP1 functions as a constitutively activated member of the tumour necrosis factor receptor (TNFR) superfamily activating a number of signalling pathways in a ligand-independent manner via engagement with TNFR-associated factors (TRAFs; Kilger et al., 1998; Mosialos et al., 
1995). The LMP1 protein activates a number of downstream signalling pathways (e.g. NF- $\kappa B$ (necrosis factor $\kappa B$ ), ERK-

MAPK (extracellular signal-regulated kinase/mitogen-activated protein kinase), phosphatidylinositol 3-kinase, activin A and/or TGF $\beta$ ) that contribute to the many growth-promoting and phenotypic consequences of LMP1 expression including the induction of various antiapoptotic and cytokine genes, influencing cell motility (Morris et al., 2009; 2016). Activation of these signalling pathways is essential for in vitro immortalization of B cells by EBV, and is likely to contribute to the initiation of EBV-associated malignancies in vivo.

The gene encoding LMP2 yields two distinct proteins, LMP2A and LMP2B. LMP2A can drive the proliferation and survival of $\mathrm{B}$ cells in the absence of signalling through the B-cell receptor (BCR). It is also able to prevent the inappropriate activation of the EBV lytic cycle. A modulatory role for LMP2B in regulating LMP2A function has been suggested (Longnecker, 2000). The precise contribution of LMP2A/B to the pathogenesis of NPC remains unknown. These encode a set of miRNAs (micro RNAs; BARTs) which are evolutionarily conserved and have been found to play a role in modulating LMP1 expression (Pfeffer et al., 2004). EBV also encodes 25 viral precursor miRNAs within its genome that are expressed during both lytic and latent infection. The virus also induces the expression of some cellular oncogenic miRNAs, including miR-155, miR-146a and miR-21, amongst others, that facilitate persistence of latently infected cells (Skalsky, 2017). BARF1 is also expressed from this region of the EBV genome and encodes a secreted protein with oncogenic activity (Seto et al., 2008).

Although, the biological signals triggering productive infection are unknown, it widely acknowledged that viral reactivation often precedes disease onset, and there is some evidence in elite athlete cohorts to suggest that high intensity exercise and the subsequent stress on the immune system and T cell exhaustion this causes may trigger EBV lytic replication (Gleeson et al., 2002; 2017).

In vitro, viral replication can be chemically induced in latently infected B cells by phorbol esters, sodium butyrate or treatment with anti-immunoglobulin-G (anti-IgG). Superinfection or transfection with the BZLF1 (BamHIZ-encoded protein 1) activation gene will also induce a fraction of the cells into productive replication (Countryman and Miller, 1985), but there is no permissive experimental cell system for EBV growth or virus quantitation.

Unlike EBV's extensively spliced gene transcripts expressed during latency, the lytic cycle gene transcripts are very compact, with relatively small introns. In productive infection, the 'immediate early' class of genes is the first to be expressed, so designated because their transcription is not dependent on de novo protein synthesis. Lytic induction triggers a cascade of gene expression of both early viral genes and cellular genes, comprising the viral replicative cycle. Overexpression of the BZLF1 protein (also known as Z, Zta, EB1 and Zebra) in lymphoid cells is alone sufficient to disrupt 
1 latency by transactivating an array of viral promoters containing Z-responsive elements. In epithelial cells, the BRLF-1 gene

2 product by itself can readily trigger the lytic cycle. Recent evidence suggests that LMP1 can promote efficient lytic

3 reactivation in EBV-infected epithelial cells by augmenting expression of the immediate early proteins BZLF1 and BRLF1,

4 and that LMP1 expression itself is induced by epithelial cell differentiation via the transcription factors KLF4 and BLIMP1

5 (Nawandar et al., 2017).

6 The 'early' genes, whose expression is triggered by the products of the 'immediate early' genes, are all involved with viral

7 DNA replication. These early antigens include a viral DNA polymerase, processivity factor, helicase, primase,

8 ribonucleotide reductase, thymidine kinase and other DNA-binding proteins that aid in replication. Because they are unique

9 to viral DNA replication, several of them are useful targets for antiviral drugs. Lytic replication of unit-length linear

10 genomes is initiated at two copies of ori-lyt (Figure $2 \mathrm{~b}$ ), which like the SV40 papovavirus origin, contains promoter and enhancer regions. Following viral DNA synthesis, the early gene products are thought to activate the 'late' or structural genes. To complete the cycle, nucleocapsids are assembled and enveloped virus particles are released by cell lysis. See also

\section{Treatment and Control}

Therapeutic approaches are mostly hypothetical compared to the effectiveness of antiviral therapy for several other human herpesviruses. A number of drugs inhibit EBV replication in cell culture, including the pyrophosphate analogues phosphonoformic acid (PFA) and phosphonoacetate (PAA), and the nucleoside analogues ara-A, ganciclovir, acyclovir, FIAC (1-(2'-deoxy-2'-fluoro-beta-D-arabinofuranosyl)-5-iodocytosine) and HPMPC ((S)-1-(3-hydroxy-2phosphonylmethoxypropyl)cytosine), all of which inhibit the virus-encoded DNA polymerase. Acyclovir suppresses viral replication in vivo, but it has been largely ineffective as a treatment for IM in clinical trials, possibly because most of the symptoms of IM are caused by secondary immunopathological responses. Success in treatment of IM will probably hinge on earlier diagnosis and use of higher drug dosage, which can be achieved with an acyclovir prodrug. Most EBV-infected tumour cells are latently infected, and would therefore be unaffected by any antiviral agents that inhibit viral replication and virus production. The only EBV disease which clearly responds to antiviral therapy is OHL, perhaps because it is an acute disease based almost entirely on active viral replication and not latent infection (Israel and Kenney, 2003). See also Antiviral Drugs

Demethylating agents such as 5'-azacytidine are able to derepress lytic, as well as potentially immunogenic, latent genes and are currently in early stage clinical trials in patients with NPC, HL and AIDS-associated lymphoma (Chan et al., 2004). Hydroxyurea is a chemotherapeutic agent that can induce loss of EBV episomes and has shown some clinical efficacy in patients with EBV-positive AIDS-related central nervous system (CNS) lymphoma. Other approaches are based on the more specific targeting of individual EBV proteins using either single chain antibodies, siRNA (small interference RNA) or dominant negative molecules. The use of adoptive EBV-specific T cell therapy for the treatment of existing EBV-induced Bcell lymphomas and in the prophylactic setting has been extremely successful (Rooney et al., 1995). This approach is also 


\section{EBV vaccine update}

More direct vaccine approaches to treat patients with EBV-associated tumours or to prevent disease development are being examined including: (i) whole EBV latent antigens delivered by a virus vector or in autologous dendritic cells; (ii) peptide or polytope vaccination and (iii) prophylactic vaccination against the MA (gp350/220) to generate a protective neutralizing antibody response, which in phase 2 clinical trials reduced the rate of IM but not of virus infection (Sokal et al., $\underline{2007}$; Cohen, 2015). One promising alternative approach for an EBV vaccine is the production of virus-like particles in which several latent gene products are functionally lost, including EBNAs 2, 3A, 3B, 3C, LMP1 and BZLF1, and which also lacks the TR packaging element required for packaging viral DNA (Ruiss et al., 2011). The resulting virus-like particles do not possess detectable EBV DNA, yet vaccination of mice with these particles still induced EBV-neutralising antibodies and cellular immune responses. Since EBV is associated with nearly 200,000 new malignant diagnoses per annum worldwide, there is a strong case to continue pursuing an EBV vaccine to prevent these and other EBV-associated diseases, with great potential for economic and societal benefit.

\section{Future Perspectives}

EBV was discovered over 40 years ago and its DNA was fully sequenced in 1984. Much work has contributed to the unequivocal identification of EBV as oncogenic in humans and it remains the most efficient agent for transforming human cells in vitro. In recent years, the nature of the interaction of EBV with the immune host has become clearer illustrating the complex mechanisms that the virus exploits to persist in the memory B cell pool. The development of more efficient in vitro systems for studying EBV infection and replication in different cell types will help to unravel the complex interplay between the virus and the cell. The use of EBV recombinants continues to shed light on the role of latent genes in the transformation process and on the requirements for the efficient production of progeny virus. Understanding the host cell:virus interaction will be dependent on the generation of appropriate in vitro models particularly systems which allow a more detailed understanding of the tumour microenvironment and the role of the local cytokine milieu. There are many interesting aspects of EBV that remain to be understood including the role of virus-encoded miRNAs and the possible contribution of lytic cycle antigens to virus persistence and oncogenesis. The challenge will be to exploit these new mechanistic insights both to gain a better understanding of EBV infection in vivo and to develop novel therapies for treating virus-associated disease.

\section{End Notes}

Based in part on the previous version of this Encyclopedia of Life Sciences (ELS) article, Epstein-Barr Virus by Lawrence S Young, Nancy S Sung, Joseph S Pagano.

\section{Glossary}

\section{APOBEC}


“Apolipoprotein B mRNA editing enzyme, catalytic polypeptide-like” - A family of cytidine deaminase enzymes involved in generating protein diversity via mRNA editing.

\title{
BARF1
}

\section{BART}

A protein encoded by the BamHIA region of the EBV genome.

\begin{abstract}
Abundant RNA transcripts encoded by the BamHIA region of the EBV genome that BZLF1 produce miRNAs.
A protein encoded from the BamHI $\mathrm{Z}$ region of the $\mathrm{EBV}$ genome which is an immediate early protein responsible for the switch between latent and lytic infection.

\section{Cell transformation}

A change to a state of unrestricted growth in culture, resembling the tumourigenic condition.

\section{Clonal tumour}

\section{EBNA}

Malignancy in which all cells share a single common genetic ancestor.

\section{Episome \\ Epstein-Bar virus-encoded nuclear antigen. \\ A circular extrachromosomal DNA element which can associate with the \\ chromosomal DNA of the host.}

\section{Latency}

\section{LMP making virions. \\ Lytic infection}

The phase in the viral life cycle in which the virus persists in the host cell without

The phase in the viral life cycle in which virions are produced and released, miRNA destroying the host cell.

Small noncoding RNA molecules that posttranscriptionally control gene expression.
Terminal repeats Repetitive units of DNA sequence situated at the ends of a linear viral genome.

\section{References}

Babcock GJ, Decker LL, Volk M and Thorley-Lawson DA (1998) EBV persistence in memory B cells in vivo. Immunity 9: 395-404.

Baer, R., Bankier, A. T., Biggin, M. D., Deininger, P. L., Farrell, P. J., Gibson, T. J., . . 
1 Seguin, C. (1984). DNA sequence and expression of the B95-8 epstein-barr virus genome.

2 Nature, 310(5974), 207-211.

3 Ballard, A. J. (2015). Epstein-barr virus infection is equally distributed across the invasive 4 ductal and invasive lobular forms of breast cancer. Pathology, Research and Practice, $5 \quad$ 211(12), 1003-1005.

6 Burkitt D (1962) Determining the climatic limitations of a children's cancer common in 7 Africa. British Medical Journal 2: 1019-1023.

8 Chan ATC, Lo YMD, Zee B et al. (2002) Plasma Epstein-Barr virus DNA and residual 9 disease after radiotherapy for undifferentiated nasopharyngeal carcinoma. Journal of the $10 \quad$ National Cancer Institute 94: 1614-1619.

11 Chan AT, Tão Q, Robertson KD et al. (2004) Azacitidine induces demethylation of the Epstein-Barr virus genome in tumors. Journal of Clinical Oncology 22: 1373-1381.

13 Chen, J., Jardetzky, T. S., \& Longnecker, R. (2016). The cytoplasmic tail domain of epstein14 barr virus $\mathrm{gH}$ regulates membrane fusion activity through altering $\mathrm{gH}$ binding to $\mathrm{gp} 42$ and 15 epithelial cell attachment. Mbio, 7(6), 10.1128/mBio.01871-16.

Cohen, J. I. (2015). Epstein-barr virus vaccines. Clinical \& Translational Immunology, 4(1), 17 e32.

18 Countryman J and Miller G (1985) Activation of expression of latent Epstein-Barr 19 herpesvirus after gene transfer with a small cloned subfragment of heterogeneous DNA.

20 Proceedings of the National Academy of Sciences of the USA 82: 4085-4089.

Dalla-Favera R, Bregni M, Erikson J et al. (1982) Human c-myc oncogene is located on the region of chromosome 8 that is translocated in Burkitt's lymphoma cells. Proceedings of the National Academy of Sciences of the USA 77: 2999-3003.

Epstein MA, Achong BG and Barr YM (1964) Virus particles in cultured lymphoblasts from Burkitt's lymphoma. Lancet $\boldsymbol{i}$ : 702-703.

Fuentes-Panana, E. M., Larios-Serrato, V., Mendez-Tenorio, A., Morales-Sanchez, A., Arias, C. F., \& Torres, J. (2016). Assessment of epstein-barr virus nucleic acids in gastric but not in breast cancer by next-generation sequencing of pooled mexican samples. Memorias do Instituto Oswaldo Cruz, 111(3), 200-208. 
1 Gleeson, M., Pyne, D. B., Austin, J. P., Lynn Francis, J., Clancy, R. L., McDonald, W. A., \& 2 Fricker, P. A. (2002). Epstein-barr virus reactivation and upper-respiratory illness in elite 3 swimmers. Medicine and Science in Sports and Exercise, 34(3), 411-417.

4 Gleeson, M., Pyne, D. B., Elkington, L. J., Hall, S. T., Attia, J. R., Oldmeadow, C., . .

5 Callister, $R$. (2017). Developing a multi-component immune model for evaluating the risk of 6 respiratory illness in athletes. Exercise Immunology Review, 23, 52-64.

7 Greenspan JS, Greenspan D, Lennette ET et al. (1985) Replication of Epstein-Barr virus 8 within the epithelial cells of oral "hairy" leukoplakia, an AIDS-associated lesion. New 9 England Journal of Medicine 313: 1564-1571.

zur Hausen H, Schulte-Holthausen H, Klein G et al. (1970) EBV DNA in biopsies of Burkitt tumors and anaplastic carcinomas of the nasopharynx. Nature 228: 1056-1058.

Henle $G$, Henle $W$ and Diehl V (1968) Relation of Burkitt's tumor-associated herpes-type virus to infectious mononucleosis. Proceedings of the National Academy of Sciences of the USA 59: 94-101.

Hu, H., Luo, M. L., Desmedt, C., Nabavi, S., Yadegarynia, S., Hong, A., . . Wulf, G. M. (2016). Epstein-barr virus infection of mammary epithelial cells promotes malignant transformation. Ebiomedicine, 9, 148-160.

Israel BF and Kenney SC (2003) Virally targeted therapies for EBV-associated malignancies. Oncogene 22: 5122-5130.

Jenkins, P. J., \& Farrell, P. J. (1996). Are particular epstein-barr virus strains linked to disease? Seminars in Cancer Biology, 7(4), 209-215.

Kelly G, Bell A and Rickinson A (2002) Epstein-Barr virus-associated Burkitt lymphomagenesis selects for downregulation of the nuclear antigen EBNA2. Nature Medicine 8: $1098-1104$.

Kilger E, Kieser A, Baumann M and Hammerschmidt W (1998) Epstein-Barr virus-mediated $B$-cell proliferation is dependent upon latent membrane protein 1, which simulates an activated CD40 receptor. EMBO Journal 17: 1700-1709.

Labrecque, L. G., Barnes, D. M., Fentiman, I. S., \& Griffin, B. E. (1995). Epstein-barr virus in epithelial cell tumors: A breast cancer study. Cancer Research, 55(1), 39-45. 
Lee, E. S., Locker, J., Nalesnik, M., Reyes, J., Jaffe, R., Alashari, M., . . Dickman, P. S. (1995). The association of epstein-barr virus with smooth-muscle tumors occurring after organ transplantation. The New England Journal of Medicine, 332(1), 19-25.

Levitskaya J, Sharipo A, Leonchiks A, Ciechanover A and Masucci MG (1997) Inhibition of ubiquitin/proteasome-dependent protein degradation by the Gly Ala repeat domain of the Epstein-Barr virus nuclear antigen 1. Proceedings of the National Academy of Sciences of the USA 94: 12616-12621.

Ling PD, Rawlins DR and Hayward SD (1993) The Epstein-Barr virus immortalizing protein EBNA-2 is targeted to DNA by a cellular enhancer-binding protein. Proceedings of the National Academy of Sciences of the USA 90: 9237-9241.

Lo KW and Huang DP (2002) Genetic and epigenetic changes in nasopharyngeal carcinoma. Seminars in Cancer Biology 12: 451-462.

Longnecker R (2000) Epstein-Barr virus latency: LMP2, a regulator or means for EpsteinBarr virus persistence? Advances in Cancer Research 79: 175-200.

Mazouni, C., Fina, F., Romain, S., Ouafik, L., Bonnier, P., Brandone, J. M., \& Martin, P. M. (2011). Epstein-barr virus as a marker of biological aggressiveness in breast cancer. British Journal of Cancer, 104(2), 332-337.

McClain, K. L., Leach, C. T., Jenson, H. B., Joshi, V. V., Pollock, B. H., Parmley, R. T., . . . Murphy, S. B. (1995). Association of epstein-barr virus with leiomyosarcomas in young people with AIDS. The New England Journal of Medicine, 332(1), 12-18.

Morris, M. A., Dawson, C. W., Laverick, L., Davis, A. M., Dudman, J. P., Raveenthiraraj, S., . . Y Young, L. S. (2016). The epstein-barr virus encoded LMP1 oncoprotein modulates cell adhesion via regulation of activin A/TGFbeta and betal integrin signalling. Scientific Reports, 6, 19533.

Morris, M. A., Dawson, C. W., \& Young, L. S. (2009). Role of the epstein-barr virus-encoded latent membrane protein-1, LMP1, in the pathogenesis of nasopharyngeal carcinoma. Future Oncology (London, England), 5(6), 811-825.

Mosialos G, Birkenbach M, Yalamanchili R et al. (1995) The Epstein-Barr virus transforming protein LMP1 engages signaling proteins for the tumor necrosis factor receptor family. Cell 80: 389-399.

Nawandar, D. M., Ohashi, M., Djavadian, R., Barlow, E., Makielski, K., Ali, A., . . Kenney, 
1 S. C. (2017). Differentiation-dependent LMP1 expression is required for efficient lytic EBV

2 reactivation in epithelial cells. Journal of Virology, doi:JVI.02438-16 [pii]

3 Pfeffer S, Zavolan M, Grasser F et al. (2004) Identification of virus-encoded microRNAS.

4 Science 304: 734-736.

5 Raab-Traub N and Flynn K (1986) The structure of the termini of the Epstein-Barr virus as a

6 marker of clonal cellular proliferation. Cell 47: 883-889.

7 Rooney CM, Smith CA, $\mathrm{Ng} \mathrm{CY}$ et al. (1995) Use of gene-modified virus-specific $T$

8 lymphocytes to control Epstein-Barr-virus-related lymphoproliferation. Lancet 345: 9-13.

9 Ruiss, R., Jochum, S., Wanner, G., Reisbach, G., Hammerschmidt, W., \& Zeidler, R. (2011).

10 A virus-like particle-based epstein-barr virus vaccine. Journal of Virology, 85(24), 13105 -

1113113.

Santon, A., Cristobal, E., Aparicio, M., Royuela, A., Villar, L. M., \& Alvarez-Cermeno, J. C. (2011). High frequency of co-infection by epstein-barr virus types 1 and 2 in patients with multiple sclerosis. Multiple Sclerosis (Houndmills, Basingstoke, England), 17(11), 12951300.

Seto E, Ooka T, Middeldorp J and Takada K (2008) Reconstitution of nasopharyngeal carcinoma-type EBV infection induces tumorigenicity. Cancer Research 68: 1030-1036.

Skalsky, R. L. (2017). Analysis of viral and cellular MicroRNAs in EBV-infected cells. Methods in Molecular Biology (Clifton, N.J.), 1532, 133-146.

Sokal EM, Hoppenbrouwers K, Vandermeulen C et al. (2007) Recombinant gp350 vaccine for infectious mononucleosis: a phase 2, randomised, double-blind, placebo-controlled trial to evaluate the safety, immunogenicity, and efficacy of an Epstein-Barr virus vaccine in healthy young adults. Journal of Infectious Disease 196: 1749-1753.

Tao, C., Simpson, S.,Jr, Taylor, B. V., \& van der Mei, I. (2017). Association between human herpesvirus \& human endogenous retrovirus and MS onset \& progression. Journal of the Neurological Sciences, 372, 239-249.

Tetzlaff, M. T., Nosek, C., \& Kovarik, C. L. (2011). Epstein-barr virus-associated leiomyosarcoma with cutaneous involvement in an african child with human immunodeficiency virus: A case report and review of the literature. Journal of Cutaneous Pathology, 38(9), 731-739. 
1 Thorley-Lawson DA and Allday MJ (2008) The curious case of the tumour virus: 50 years of

2 Burkitt's lymphoma. Nature Reviews. Cancer 6: 913-924.

3 Thorley-Lawson DA and Gross A (2004) Persistence of the Epstein-Barr virus and the origins of associated lymphomas. New England Journal of Medicine 350: 1328-1337.

5 Tsai, M. H., Lin, X., Shumilov, A., Bernhardt, K., Feederle, R., Poirey, R., . . Delecluse, H.

6 J. (2017). The biological properties of different epstein-barr virus strains explain their

7 association with various types of cancers. Oncotarget, 8(6), 10238-10254.

8 Wang D, Liebowitz D and Kieff E (1985) An Epstein-Barr virus protein expressed in

9 immortalized lymphocytes transforms established rodent cells. Cell 43: 831-840.

10 Yates J, Warren $N$ and Sugden $B$ (1985) Stable replication of plasmids derived from Epstein11 Barr virus in various mammalian cells. Nature 313: 812-815.

\section{Further Reading} 4: 757-768. Publishers. 251-262.

Young L, Alfieri C, Hennessy K et al. (1989) Expression of Epstein-Barr virus transformation-associated genes in tissues of patients with EBV lymphoproliferative disease. New England Journal of Medicine 32: 1080-1085.

Young LS and Rickinson AB (2004) Epstein-Barr virus: 40 years on. Nature Reviews. Cancer

Kieff E and Rickinson AB (2006) Epstein-Barr virus and its replication. In: Knipe DM and Howley PM (eds) Fields Virology. 5th edn. Philadelphia: Lippincott Williams and Wilkins

Kuppers $R$ (2005) Mechanisms of B cell lymphoma pathogenesis. Nature Reviews. Cancer 5:

Kutok JL and Wang F (2006) Spectrum of Epstein-Barr virus-associated diseases. Annual Review of Pathology: Mechanisms of Disease 1: 375-404.

Rickinson AB and Kieff E (2006) Epstein-Barr virus. In: Knipe DM and Howley PM (eds) Fields Virology. 5th edn, Philadelphia: Lippincott Williams and Wilkins Publishers. 
1 Robertson ES (ed.) (2005) Epstein-Barr Virus. Norfolk, England: Caister Academic Press.

2 Tao Q, Young LS, Woodman CB and Murray PG (2006) Epstein-Barr virus and its

3 associated cancers - genetics, epigenetics, pathobiology and novel therapeutics. Frontiers in

4 Bioscience 11: 2672-2713.

5

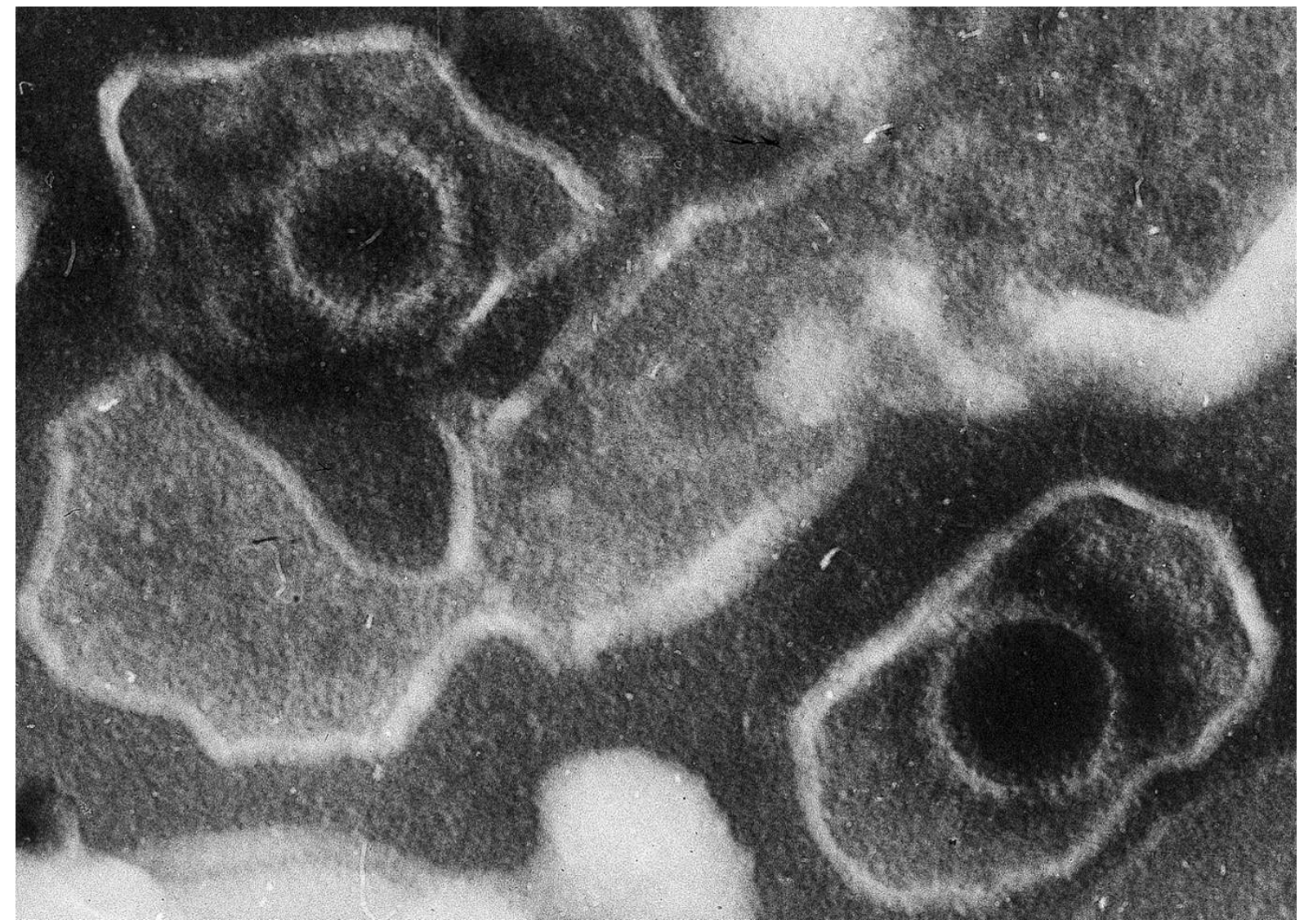

7 Figure 1. Electron micrograph of the Epstein-Barr virus virion. 
Table 1 - Diseases associated with Epstein-Barr virus (EBV) infection

\begin{tabular}{ll}
\hline Tissues affected and diseases & Role of EBV \\
\hline Lymphoid & \\
Burkitt Lymphoma & Cofactor, nonessential; clonal association ${ }^{\mathrm{a}}$ \\
B-cell immunoblastic lymphomas & Causal \\
T-cell lymphomas & Clonal association ${ }^{\mathrm{a}}$ \\
Hodgkin disease & Clonal association $^{\mathrm{a}}$, Reed-Sternberg cells infected \\
Chronic interstitial lymphocytic pneumonitis & Causal \\
Epithelial & \\
Nasopharyngeal carcinoma & Essential cofactor $_{\text {Parotid carcinoma }}$ \\
Gastric carcinoma & Clonal association $^{\mathrm{a}}$ \\
Oral hairy leukoplakia & Clonal association \\
Lymphoid and epithelial & Causal \\
Infectious mononucleosis & \\
Other & Causal \\
Leiomyosarcoma & \\
\hline
\end{tabular}

${ }^{\mathrm{a} C}$ Clonal association refers to episomes which reflect clonality of tumour cells in which they are found, with differences in the number of terminal repeats (TRs; Figure 3) characterising different 'clones'. 
Primary infection

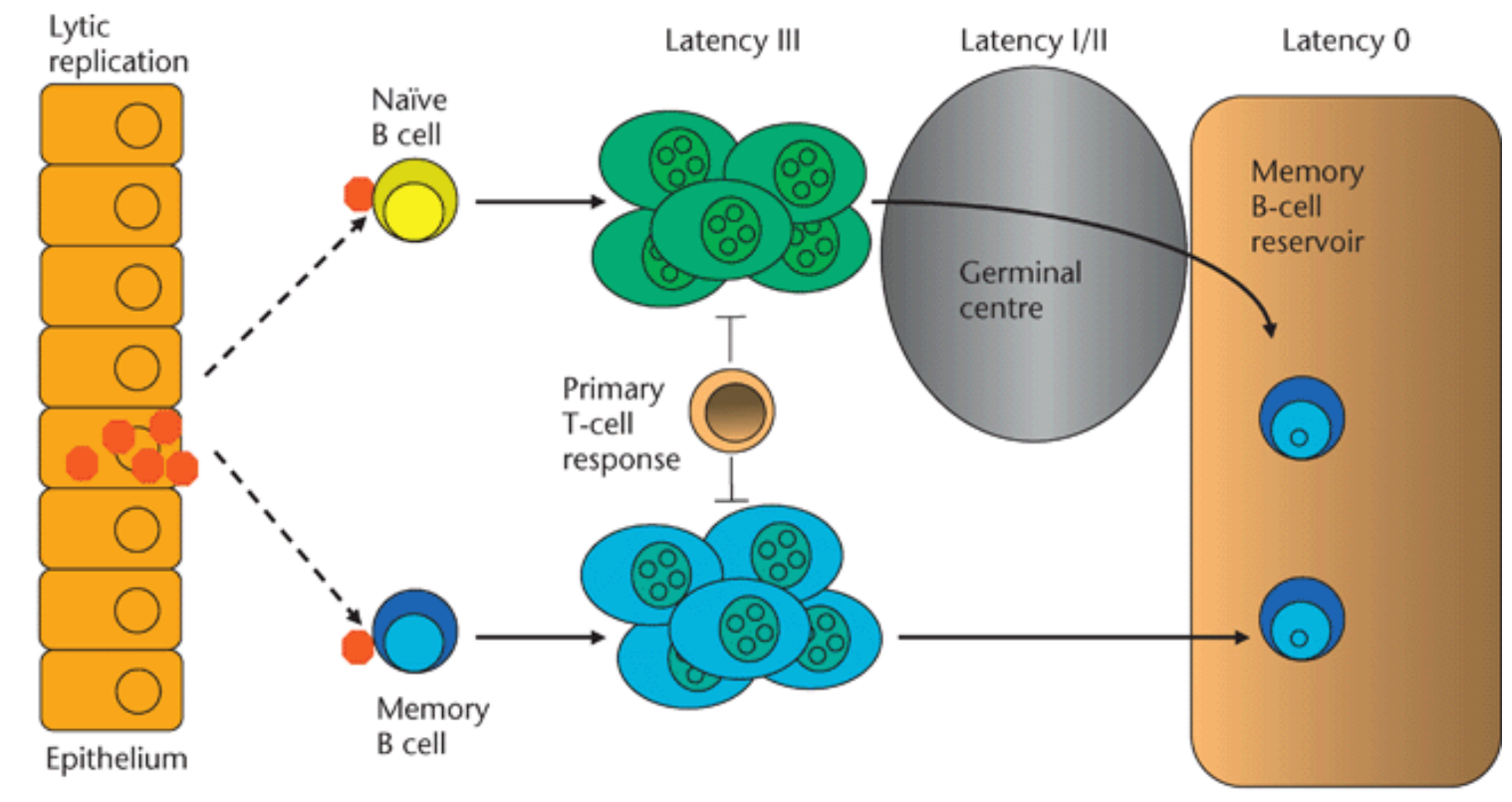

(a)

Persistent infection

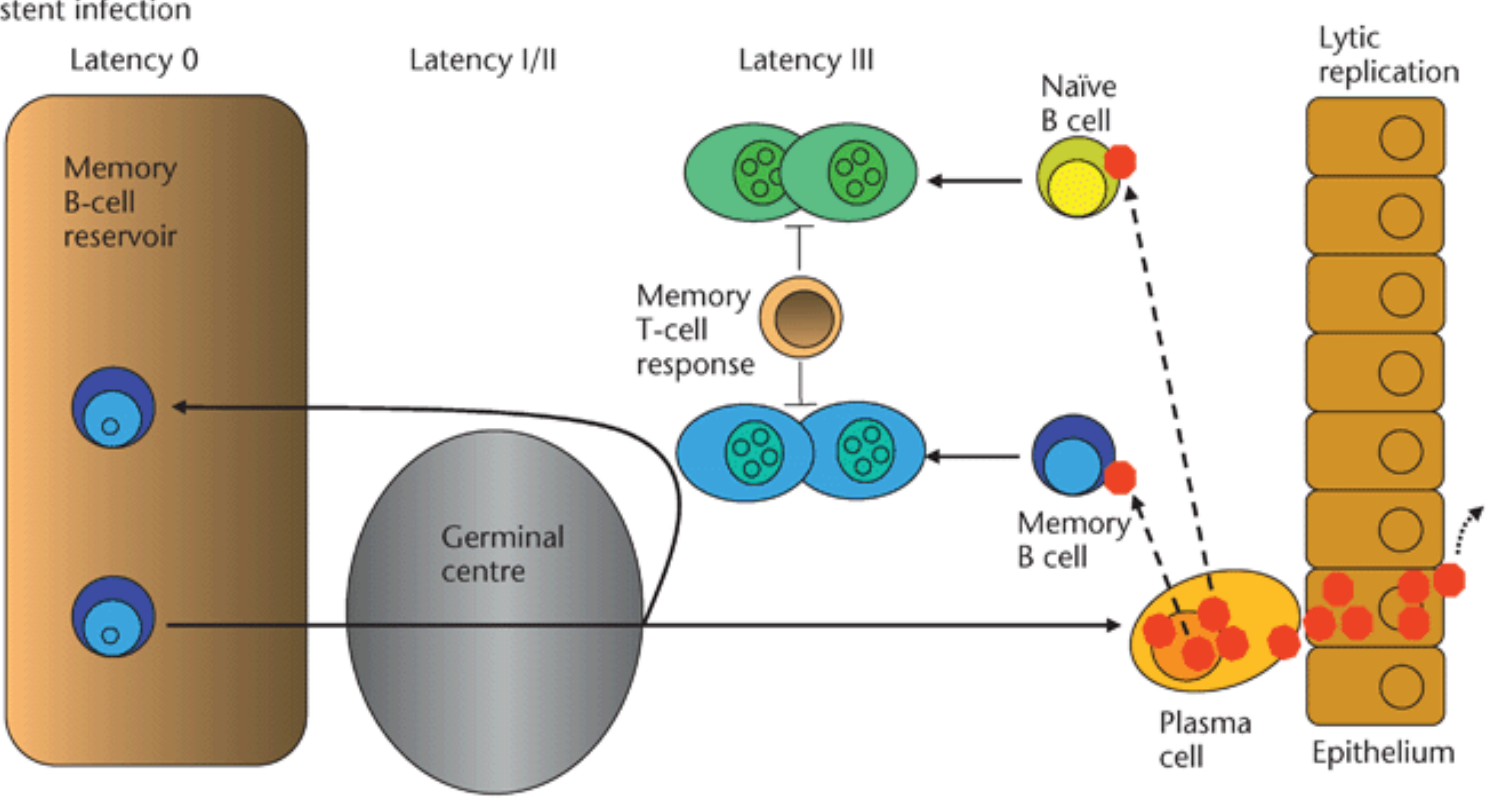

(b)

3 Figure 2. EBV primary infection and persistence. Figure showing putative in vivo

4 interactions between EBV and host cells. (a) Primary infection. EBV replicates in epithelial

5 cells and spreads to lymphoid tissues as a latent growth-transforming (latency III) infection of

6 B cells. Many infected B cells are removed by emerging EBV-specific T-cell response. Some

7 infected cells escape by downregulating EBV latent genes to establish a stable pool of resting

8 virus-positive memory B cells. (b) Persistent infection. EBV-infected memory B cells

9 become subject to the physiological controls governing memory B-cell migration and

10 differentiation. Occasional recruitment into germinal centre (GC) reactions resulting in 
1 activation of different EBV latency programmes and reentry into the memory cell reservoir or 2 plasma cell differentiation with activation of virus lytic cycle. Infectious virions then initiate 3 foci of EBV replication in epithelial cells and also new growth-transforming infection of 4 naïve and/or memory B cells. For more detailed explanation see Young and Rickinson 2004 5 and Thorley-Lawson and Gross 2004. Reprinted from Mahy BWJ and Van Regenmortel 6 MHV (2008) Epstein-Barr virus: General features. Encyclopedia of Virology, with 7 permission from Elsevier.

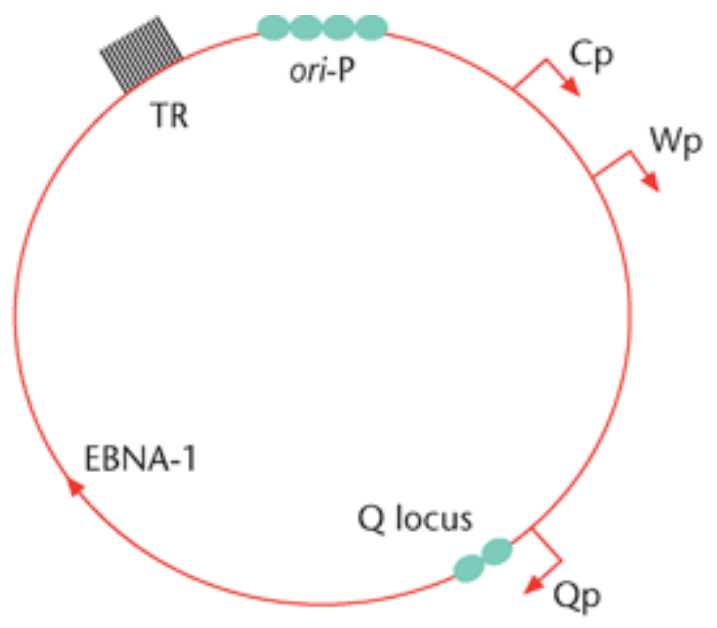

(a)

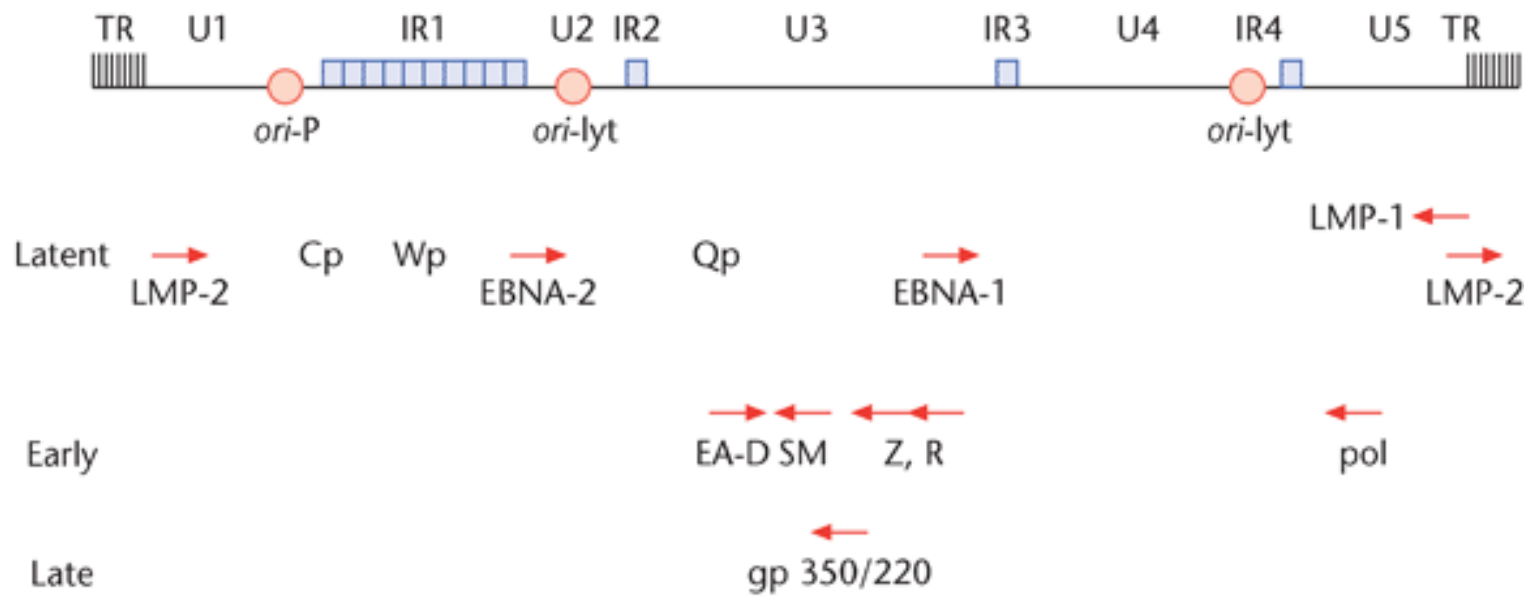

8 (b)

9 Figure 3. Epstein-Barr virus (EBV) genome forms. (a) The episome, in which the EBV 10 genome circularizes via the terminal repeats (TR), is the hallmark of EBV latency. EBNA1 is 11 the principal EBV latency protein. EBNA promoters are shown (Cp, Wp, Qp), as well as the 12 EBNA1 open reading frame (triangle). Shaded circles represent the two areas of the EBV 
1 genome where EBNA1 dimers bind. Two binding sites are found in the Q locus, whereas 24

2 binding sites are found within the plasmid origin of replication (ori-P). EBNA-1 activates ori-

$3 \mathrm{P}$ and autoregulates Qp. (b) The linear EBV genome is diagnostic of productive infection.

4 The positions of terminal (TR) as well as internal direct (IR) repeat units are shown. Regions

5 of unique sequence are designated U. Latent and lytic (ori-lyt) origins of DNA replication are

6 shown as open circles. Arrows pointing rightward or leftward indicate the location and

7 direction of transcription of the coding sequences of the EBV genes mentioned in the text. 